\title{
LA ETNICIDAD ANDALUZA EN CATALUÑA: CRITERIOS PARA UNA TIPOLOGIA
}

\author{
Emma MARTIN DIAZ*
}

\section{INTRODUCCION}

Uno de los principales problemas que plantea el estudio de la etnicidad de los inmigrantes en el marco de la sociedad de acogida es la escasez de modelos teóricos ya elaborados sobre este tema. Los datos obtenidos a lo largo de nuestra investigación sobre las redes sociales y la identidad de los andaluces en Barberá del Vallés ${ }^{1}$ y las diferentes investigaciones sobre etnicidad y nacionalismo que se están llevando a cabo en el seno del Estado español son las bases del modelo que vamos a desarrollar a continuación.

Las migraciones en el interior del Estado español son producto de las diferentes situaciones específicas en las que se encuentran los distintos pueblos existentes en su seno y que traen como consecuencia que haya un fuerte desequilibrio entre zonas escasamente desarrolladas y otras con elevado nivel de desarrollo. En buena parte, la situación es producto de las condiciones concretas en las que los diversos territorios se incorporaron al Estado español, pero de ninguna manera debe olvidarse que éste juega un papel fundamental en la existencia de estos desequilibrios en la medida en que contribuye a reforzar la situación existente.

El fuerte desequilibrio regional característico del Estado español es el marco en el que hay que situar el fenómeno migratorio, pero no es el único hecho a tener en cuenta. La emigración interior perfila unos marcos de confrontación interétnica muy complejos e interesantes, que hacen necesario un análisis detallado de todos sus aspectos y su complejidad mediante un aparato conceptual adecuado. Si no tenemos en cuenta que en el fenómeno migratorio intervienen diversas realidades a diferentes niveles, que mantienen entre sí una interde-

* Profesora Asociada de Antropología Cultural. Universidad de Sevilla.

1. Esta investigación fue el objeto de mi tesis doctoral, el trabajo de campo tuvo lugar durante los años 1985-86. 
pendencia estructural históricamente delimitable, corremos el riesgo de analizarlo de una forma descontextualizada. Este tipo de análisis conllevaría que no se puedan establecer más que consideraciones muy generales -como que las migraciones suponen un trasvase de mano de obra de las zonas subdesarrolladas a las más desarrolladas o que aquéllos que emigran pertenecen a los estratos más bajos de la sociedad-y otras referentes a determinados aspectos comportamentales y de valores, que tienen en cuenta la «realidad del emigrante» descontextualizándola de los procesos culturales en los que éste se encuentra inmerso. Así, bajo el pretexto de realizar un enfoque holístico del fenómeno migratorio se cae en un reduccionismo en el que sólo se tienen en cuenta aquéllas actitudes que no hacen referencia a la etnicidad de los colectivos relacionados en este proceso, limitándose a señalar lo que tienen en común los distintos fenómenos migratorios, se producen en el marco que se produzcan.

\section{EL MARCO TEORICO DE LA ETNICIDAD}

Por otra parte, una constante en muchas investigaciones realizadas sobre el tema de la etnicidad es la concepción de lo etnico como un todo homogéneo. Así, se olvida que agrupados entorno a este concepto se encuentran conceptualizaciones tan dispares como tribu, minoría étnica, pueblo región, país, nacionalidad y nación. Uno de los hechos que puede explicar la confusión existente en torno al concepto de etnicidad es que en las investigaciones sobre los fenómenos étnicos se suelen obviar o simplificar excesivamente las causas que los generan, aquéllas por las que un grupo social persiste en reivindicar o hacer significativas sus diferencias.

En este sentido, resulta imposible definir a los grupos étnicos en base a la posesión de una misma serie de rasgos, pues éstos varían para cada grupo. Así, aunque la presencia de rasgos diferenciales en un grupo humano es condición necesaria para la existencia de un grupo étnico, no es la base que explica su existencia como tal. Estos rasgos son los marcadores de la etnicidad, pero son precisos catalizadores que los pongan en movimiento. Por ello, aunque resulta de gran interés conocer cuáles son los rasgos que cada grupo social considera como marcadores de la diferenciación, es mucho más importante averiguar las causas por las que un grupo social asume esta conciencia diferenciadora. Se trata de comprender porqué y cómo se genera y mantiene la conciencia de diferenciación que supone la identidad de un grupo concreto. En este proceso de contextualización de los diferentes grupos étnicos existentes resulta de fundamental importancia analizar la existencia de una realidad macrosocial, la del Estado, que determina en gran medida las relaciones entre los diferentes grupos étnicos existentes en su seno. 
La inclusión en los estudios sobre etnicidad del marco estatal permite distinguir una serie de fenómenos estructuralmente diferentes: los movimientos coloniales, las minorías étnicas, los regionalismos y los nacionalismos ${ }^{2}$.

Los movimientos coloniales presentan dos características fundamentales: por un lado su heterogeneidad cultural, y por otro el hecho de que los diversos grupos étnicos se unen de una manera coyuntural en su lucha contra la metrópoli. Independientemente de la forma que adopten, estos movimientos tienen en común el hecho de que el dominio de clase aparece bajo la forma de conflicto étnico.

Una de las principales características de las minorías étnicas es su relación con la formación de grandes concentraciones urbanas en el proceso de industrialización. Estas concentraciones atraen a inmigrados de diferentes grupos étnicos, dando lugar a una gran heterogeneidad cultural. Junto a ella, se produce una división del trabajo basada en las divisiones étnicas, de forma que los distintos grupos étnicos tienen una diferente posición en el acceso y control de los recursos existentes. Estos hechos determinan que las minorías étnicas opten por defender el reconocimiento de su especificidad cultural con el objeto de defender $\longrightarrow$, mucho más frecuentemnete, lograr- una posición favorable en el reparto de estos recursos.

Los movimientos regionalistas dentro de los estados se caracterizan por no poner en tela de juicio el tipo de integración que su grupo y su territorio tienen en el seno de estas formaciones. A lo sumo, intentan conseguir una descentralización administrativa y una autonomía cultural. De esta forma, se contribuye a reforzar la pretensión del Estado de presentarse como única Nación-Estado, negando que los grupos étnicos puedan tener en su interior otra existencia que la regional.

Como señala I. MORENO, para que existan movimientos nacionalistas es imprescindible que «una clase o alianza de clases funda su conciencia de clase con la conciencia nacionalista, teniendo como voluntad explícita la afirmación de la identidad no sólo cultural, sino también política de su pueblo» ${ }^{3}$. Los movimientos nacionalistas cuestionan el modelo de integración económico y político que el grupo étnico y su territorio tienen dentro del Estado, negando que las diferencias existentes se limiten exclusivamente a determinadas particularidades culturales, y propugnando que sólo en el grupo reside la legitimidad para decidir sobre sus asuntos y sobre el tipo de relaciones que se establecen con los demás grupos étnicos.

2. El modelo teórico procede de la investigación que sobre la identidad étnica y los procesos migratorios en Cataluña han realizado un grupo de antropólogos catalanes bajo la dirección de los doctores Comas, Frigolé, Juliano, Provansal y Pujadas.

3. Moreno, I.: «Etnicidad, conciencia de etnicidad y movimientos nacionalistas: el caso andaluz». Revista de Estudios Andaluces, núm. 5, pp. 13-38, 1985. 


\section{EL CASO DEL ESTADO ESPAÑOL}

En el marco concreto del Estado español, a la tradicional problemática de los regionalismos y/o nacionalismos hay que añadir un fenómeno histórico relativamente reciente, pero de gran trascendencia: las migraciones en masa desde los países y regiones menos desarrolladas del Estado español -como Andalucía - a las zonas industrializadas de éste —como Cataluña-. Al ser España un estado pluriétnico, las relaciones que se establecen entre los distintos grupos étnicos existentes en su territorio son variadas y complejas, pudiendo adoptar diferentes manifestaciones según el contexto histórico, socioeconómico y político en el que dichos grupos interactúan. Así, puede darse el caso de que una parte importante de un grupo étnico adopte en el interior de su territorio una determinada manifestación de su etnicidad y en otro territorio del mismo Estado adopte una forma distinta, al variar el contexto en el que está inmerso. Nuestro objetivo principal es averiguar la forma específica que adquiere la manifestación de la etnicidad andaluza en Cataluña. Para ello, vamos a utilizar un modelo teórico que, aunque basado en los anteriormente citados, modifica parcialmente éstos, adaptándolos al problema concreto a investigar. Así, consideramos que los andaluces en Cataluña pueden insertarse en las siguientes categorías:

\section{MODELOS DE INSERCION DE LOS ANDALUCES EN CATALUÑA}

Podemos considerar a los andaluces en Cataluña como:

"Minoría regional». En este caso concreto, los andaluces en Cataluña se limitarían a expresar sus particularidades culturales, pero sin poseer una clara conciencia de que éstas los definen como un grupo específico. Se las considera como manifestaciones integradas en el conjunto de manifestaciones culturales del Estado español, como una variante de éstas. Por supuesto, no se cuestiona el modelo de integración económica y política de los grupos étnico-territoriales en el Estado, por lo que los problemas de los inmigrantes andaluces en Cataluña serían comunes a los del conjunto de los inmigrantes provenientes de otras partes del Estado, y se reducirían - una vez que se aceptara, sin reconocimiento especial, su particularidad cultural- a un problema de clase.

Otra de las posibilidades es que los andaluces formen una «minoría étnica». En el caso concreto de Cataluña, ello supondría que a la percepción de la indudable heterogeneidad cultural que ha traido consigo la masiva inmigración experimentada, se uniese una división del trabajo que siguiese las líneas de 
la división étnica, de forma que los autóctonos ocupen las posiciones más favorables de la escala social. En este caso, la reivindicación de la especificidad cultural de los andaluces estaría estrechamente conectada con el objetivo de conseguir ocupar las mismas posiciones que la población autóctona.

La tercera posibilidad es que los andaluces en Cataluña formen una «minoría nacional», que hayan tomado conciencia no sólo de la identidad cultural de Andalucía, sino de su identidad política, diferenciada del resto del Estado. Una minoría nacional es aquélla que defiende que el derecho al control de los recursos económicos y las decisiones políticas debe recaer en el grupo étnico, es decir, que el grupo étnico y su territorio forman una nación, oponiéndose por tanto a la existencia única del Estado-nación.

Con esta tipología no pretendemos afirmar que la totalidad de los andaluces en Cataluña deben insertarse forzosamente en una de estas tres categorías. Esto sería caer en un esencialismo que se aleja considerablemente de nuestros objetivos. Las categorías establecidas deben ser entendidas como modelo para el estudio de la etnicidad andaluza en Cataluña, y no como realidades cerradas e incontrovertibles.

\section{LOS ESTUDIOS SOBRE LA EMIGRACION ANDALUZA EN EL CONTEXTO DEL ESTADO ESPAÑOL}

Como hemos señalado, la mayoría de los estudios que tienen como objeto el fenómeno migratorio tienden a desvincularlo del contexto concreto en el que éste se inscribe. A lo sumo, se consideran aspectos generales que están en la base de todo fenómeno migratorio, se produzca donde se produzca. La esterilidad de este planteamineto es fácilmente observable en las conclusiones finales de estos trabajos, que la mayoría de las veces son auténticas tautologías.

En el caso concreto de las migraciones interiores del Estado español, la falta de contextualización del fenómeno lleva consigo la utilización de los conceptos referenciales de comunidad de origen y comunidad de acogida. De esta forma, al no tener en cuenta el marco concreto en el que las comunidades se inscriben, la atención del investigador ha de centrarse necesariamente sólo en los procesos de cambio del mundo rural al mundo urbano y de la actividad agrícola a la industrial, procesos que están presentes en la práctica totalidad de los procesos migratorios actuales casi en cualquier parte del mundo y que es necesario analizar, pero que no constituyen la totalidad del fenómeno y que son tomados como si lo fueran. Este enfoque es la causa de que la mayoría de los trabajos desemboquen en una serie de estériles generalizaciones e incluso mixtificaciones del fenómeno migratorio. 
En lo que se refiere a la inmigración andaluza a Cataluña, el análisis se realizaría sin apenas tener en cuenta las realidades culturales de los grupos étnicos que entran en contacto en este proceso. De esta forma, se pasa del marco de los pueblos y ciudades de procedencia de los inmigrantes a los lugares en los que éstos se establecen, para concluir que las migraciones en el interior del Estado español son debidas al escaso desarrollo de algunas zonas y la industrialización de otras, pero sin analizar el marco concreto en el que se inscriben estos desequilibrios regionales a los que se hace alusión y por tanto, sin tener en cuenta el complejo marco de relaciones interétnicas que se establece.

Los análisis que parten de este enfoque tienden a hacer hincapié en el carácter rural de los emigrantes identificando lo rural con lo arcaico, oponiendo lo «tradicional» a lo industrial, e identificando a éste con lo moderno. Así, los inmigrantes, una vez establecidos en los Iugares de destino, estarían sujetos a un proceso de «modernización» que tendería a modificar su universo de valores y sus pautas culturales de origen, que dejarían de ser operativas en el nuevo contexto. Sin embargo, este análisis, basado en la oposición citada, es siempre simplista y, sobre todo, tiene poca validez en el caso concreto de los andaluces en Cataluña.

La identificación rural-tradicional y su oposición a lo urbano-moderno parte de la visión de un mundo rural anclado en unas estructuras agrarias fosilizadas y escasamente competitivas, anteriores al establecimiento del modo de producción capitalista o en las primeras fases de este. Ante esta situación, la población más emprendedora de los núcleos rurales comenzaría a emigrar como producto de un deseo de mejorar su condiciones de vida y de insertarse en el mundo moderno e industrial, en el contexto de un modo de producción plenamente capitalista. Quienes aplican este enfoque el caso andaluz no tienen en cuenta que las estructuras agrarias andaluzas se insertan plenamente, y no sólo desde época reciente, dentro del modo de producción capitalista, y que es la modernización producida por la introducción en las últimas décadas de nuevos cultivos y de maquinaria agrícola la causa detonante del excedente de mano de obra asalariada y de la crisis de las pequeñas explotaciones y, por tanto, de la emigración andaluza. Así, pues, la emigración andaluza no puede analizarse desde el punto de vista de la crisis de las estructuras agrarias tradicionales. Es más, el sector agrario andaluz, como demuestran los más recientes trabajos de los investigadores andaluces que se han ocupado de la cuestión ${ }^{4}$, es uno de los primeros en incorporar las relaciones de producción capitalistas a sus estructuras desde hace siglos.

4. Esta es la tesis de autores como Bernal, A.M., quien ha señalado en muchos de sus trabajos la importancia que adquiere para Andalucía la temprana inserción de las estructuras agrarias andaluzas en el modo de producción capitalista. 
La descontextualización a la que hemos hecho referencia no es la única simplificación en la que se incurre al analizar los fenómenos migratorios. En el caso concreto de Cataluña, el error más frecuente de los abundantes trabajos sobre el tema de la inmigración consiste, en virtud de un proceso similar de descontextualización, en oponer catalanes e inmigrados como si fuesen dos grupos homogéneos culturalmente enfrentados. Una de las bases en las que descansa este error es la identificación que se establece entre lengua y cultura. En este caso, la división existente entre catalanoparlantes y castellanoparlantes con motivo de la utilización de una u otra lengua en la interrelación social trasciende este marco concreto para convertirse en la barrera que separa dos grupos que son percibidos como homogéneos. De esta forma se olvida que la lengua es el vehículo transmisor de los elemento que componen la cultura, es decir, del conjunto de prácticas, normas, valores, creencias y actitudes que la constituyen, pero no es en sí misma la cultura, aunque pueda ser el símbolo más característico de ésta.

\section{LA NECESARIA INTEGRACION DE LA INMIGRACION}

Esta visión de la sociedad catalana como compuesta por dos bloques diferentes de catalanes por un lado e inmigrantes por otro, da lugar a una serie de posturas que, partiendo de diferentes postulados, tienden a la misma conclusión: la necesidad de integración del colectivo de los inmigrantes en la sociedad catalana, o lo que es lo mismo, la necesidad de que se produzca la catalanización de los inmigrantes.

Uno de los planteamientos se basa en la consideración del peligro que supone para la sociedad catalana su división en dos bloques enfrentados entre sí: catalanes por un lado e inmigrantes por otro, o bien castellanoparlantes frente a catalanoparlantes. La solución propuesta para que no se produzca esta división es la integración de los inmigrantes, formulada en términos de la aceptación de la cultura catalana y su progresiva inserción en ella, si no de la primera generación de inmigrantes, sí de sus hijos. Esta es la postura adoptada, con diferentes matices, por la mayoría de las fuerzas políticas en Cataluña.

El segundo planteamiento se limita a negar esta división cultural de la sociedad catalana, reduciendo la inmigración a un problema de clases. Esta postura reduccionista es la adoptada mayoritariamente por los grupos de intelectuales que se reclaman marxistas, y se encuentra muy extendida en el interior de la clase obrera. Sin embargo, la aceptación de este presupuesto teórico no impide que en la realidad la existencia de difernetes grupos étnicos con distintos intereses se imponga a la teoría. 
Pese a partir de planteamientos radicalmente diferentes, ambas posturas coinciden en señalar como fin de este proceso la integración de los inmigrantes no sólo en la sociedad sino también en la cultura catalana, valorándola, desde la primera, como indispensable para cristalizar Cataluña como nación, definida a través de su cultura, o para «solucionar» el tema incómodo de las diferencias étnicas y hacer posible una más nítida lucha de clases desde la segunda. Pero en ambos casos se trataría de que desapareciera el colectivo inmigrante, al que se dota de una existencia independiente y diferenciada.

Sin embargo, nosotros consideramos que el análisis de la inmigración en Cataluña debe partir de la consideración de que ésta no puede ser analizada como constituida por un bloque homogéneo formado por los no cảtalanes que han acudido a vivir y a trabajar en Cataluña. Pese a esta característica común, la inmigración está compuesta por diferentes grupos pertenecientes a ámbitos culturales específicos, los cuales presentan un variado grado de cohesión y autoconciencia de etnicidad.

\section{LOS ANDALUCES EN CATALUÑA: ¿MINORIA «REGIONAL», «ETNICA»O «NACIONAL»?}

Para nosotros, los andaluces en Cataluña pueden adoptar diferentes posiciones que nos permiten insertarlos en una de las tres categorías que componen nuestro modelo teórico de partida: minoría regional, minoría étnica y minoría nacional. En la inclusión en una u otra categoría influirán el grado de autoconciencia de etnicidad, la percepción y utilización de los símbolos de identidad étnica y la posición que adopten acerca del derecho de los grupos étnicos a manifestarse como tales.

Así, un grupo relativamente importante de andaluces en Cataluña constituye lo que hemos denominado una minoría regional. En este sentido, sus aspiraciones se limitan a conseguir la aceptación de la expresión de su particularidad cultural. Sin embargo, dentro de este colectivo podemos distinguir dos subgrupos claramente diferenciados.

En el primer subgrupo estarían aquéllos que simplemente desean expresar, sin obstáculos de ningún tipo, determinadas manifestaciones culturales de su tierra de origen. Dentro de este subgrupo estarían aquéllos andaluces conmenos conciencia de clase, que no se plantean, además ningún otro tipo de reivindicación. Para ellos no existe, al menos de manera consciente, ninguna diferencia entre vivir en Andalucía y vivir en Cataluña, aceptando pasivamente el planteamiento de que la inmigración interior supone exclusivamente un cambio de residencia dentro del Estado. Pero, además, dentro de este subgrupo existe una minoría politizada, que contempla el creciente movimiento na- 
cionalista catalán como un peligro para la unidad del Estado. En este sentido, las reivindicaciones y expresiones de la particularidad cultural andaluza de esta minoría serían un intento de poner freno al citado movimiento nacionalista, negando que Cataluña pueda tener una existencia política diferenciada en el Estdo español. Dentro de ella estarían los promotores y fundadores de los centros culturales andaluces de la etapa de las Casas Regionales de los años sesenta - fundamentalmente militares y funcionarios de la administración estatal-, algunos andaluces pertenecientes a los estratos medio y alto y los directivos de la Federación de Entidades Andaluzas en Cataluña (FECAC) y de la desaparecida emisoria Radio Tele-Taxi.

El otro subgrupo está formado por un sector de andaluces pertenecientes a la clase obrera, que se identifican con las posiciones del marxismo más ortodoxo o, sobre todo, con los partidos estatales de izquierda. Al contrario que el subgrupo anterior, éste último no es partidario de la expresión de las manifestaciones culturales de los diferentes grupos étnicos en Cataluña, pues consideran que éstas no hacen más que retardar la necesaria integración de los inmigrantes en la sociedad y en la cultura catalanas. Esta posición se basa en el hecho de que, para los miembros de este subgrupo, los problemas de los inmigrantes son los mismos que afectan al conjunto de la clase obrera, esté donde esté. Para ellos, la inmigración supone una decisión de carácter individual y se limita exclusivamente a un cambio de ocupación y residencia. En el fondo de esta postura, y aunque de ello no sean conscientes la mayor parte de los que la mantienen, se encuentra la negación de que puedan existir distintas formaciones económico-sociales en el seno del Estado.

Aunque, a diferencia del anterior, este subgrupo no es partidario de la expresión de las manifestaciones culturales de los grupos étnicos existentes en Cataluña, una minoría formada por aquéllos que ocupan cargos de responsabilidad en los partidos citados, intenta, en la medida en que estas manifestaciones se producen, instrumentalizarlas en su propio beneficio, asistiendo y participando en los actos que se organizan, pero procurando dar a su participación un carácter regional, acorde con su postura sobre el tema. En este sentido hay que entender la postura del Ayuntamiento de Barberá de organizar, en la caseta que instaló durante la Feria de Abril de 1987, una serie de conferencias dedicadas a los diferentes colectivos de inmigrantes en Cataluña, intentando diluir el carácter exclusivamente andaluz de este acto.

La mayoría de los andaluces en Cataluña forman una minoría étnica, de acuerdo con la definición que dábamos anteriormente. O sea, un colectivo de individuos que asumen una conciencia de identidad en condiciones de gran heterogeneidad cultural. En el proceso de constitución de los andaluces en minoría étnica han influido varios factores: en primer lugar, el elevado número de inmigrantes andaluces y su concentración en barrios y pueblos donde su 
presencia es numéricamente importante e incluso mayoritaria. Es evidente que este hecho dificulta considerablemente la integración al favorecer el establecimiento de redes sociales similares a las existentes en los lugares de procedencia, las cuales, a su vez, propician el mantenimiento y reproducción de la identidad de las personas insertas en estas redes.

La evidencia de la pluralidad cultural resultante del fenómeno migratorio no es suficiente para que se produzca la presencia de minorías étnicas con conciencia de tales. Es necesario que exista además una división del trabajo que permita que unos grupos étnicos asuman unas posiciones más favorables que las demás. En este sentido, es evidente que los inmigrantes en Cataluña han venido ocupando tradicionalmente los puestos más bajos de la escala social y que su presencia ha fomentado una división en el seno de la clase obrera, permitiendo que los autóctonos pasen a desempeñar los puestos de trabajo mejor considerados socialmente, que exigen cierta cualificación, frente a una fracción, compuesta mayoritariamente por inmigrados, que ocupan los puestos de trabajo peor considerados socialmente y menos cualificados. Además, esta fracción de la clase obrera se ve considerablemente más afectada por el paro por sus propias características de escasa preparación profesional.

Somos conscientes de que en el interior de la clase obrera catalana no existe una división mecánica en dos bloques claramente diferenciados de autóctonos e inmigrantes. La situación socioeconómica de la clase obrera catalana es muy variada y tampoco se articula mecánicamente sobre el origen étnico de sus miembros. Sin embargo, esta división existe y es percibida por los andaluces en términos de etnicidad, considerando que los trabajadores autóctonos ocupan un nivel superior al de los inmigrantes. A partir de esta percepción, los factores étnicos cobran un papel primordial en las relaciones entre los diversos grupos puestos en contacto.

Aunque la mayoría de los andaluces han mantenido su identidad cultural específica durante todo el período de su inmigración, ésta no ha permanecido invariable, sino que ha asumido distintas formas. Si en una primera etapa de la inmigración los recién llegados se limitaban a reproducir las redes sociales y parentales existentes en sus lugares de origen, la posterior toma de contacto con las luchas y reivindicaciones laborales en sus respectivos centros de trabajo - en particular durante los años setenta-y la incorporación al fuerte movimiento vecinal de esta misma época, les hizo caminar junto a los catalanes en el proceso de democratización de la sociedad, que, en el caso de Cataluña, iba aparejada a la lucha por su autonomía. Sin embargo, la mayoría de los que asumieron la defensa de la autonomía catalana lo hicieron desde su propia posición de andaluces, reivindicando paralelamente la autonomía para Andalucía. La idea de formar parte de un colectivo diferente al autóctono, y con intereses específicos que defender explica que el Partido Socialista de Andalu- 
cía (PSA) consiguiera dos escaños en el Parlamento en las primeras Elecciones Autonómicas de 1980.

Posteriormente, la prolongación de la crisis económica y la normalización de la vida política - que en Cataluña lleva consigo la hegemonía de los sectores nacionalistas de centro derecha- provoca un alejamiento de los andaluces de la vida política que dificulta aún más la integración y favorece su marginación. Esta etapa se caracteriza por una eclosión de las manifestaciones culturales de carácter andaluz que suponen un auténtico trasplante cultural y que son secundadas masivamente por este grupo étnico, que patentiza de esta forma su especificidad cultural. El objetivo concreto que persigue este grupo con este comportamiento es el mismo que persiguen todas las minorías étnicas donde quiera que estén: el logro del reconocimiento de su identidad cultural diferenciada del grupo mayoritario para reivindicar una consideración social y una serie de derechos que no les son reconocidos. En este sentido hay que entender la reivindicación de que pueda verse en Cataluña la televisión autonómica andaluza.

Además de los dos grupos citados, hay un sector de los andaluces en Cataluña que forman lo que hemos caracterizado una minoría nacional. Son aquéllos que no solamente son conscientes de la identidad cultural específica de los andaluces, sino que dan un paso más y consideran que es en éstos en quienes reside el derecho al control de los recursos económicos y de las decisiones políticas, considerando que la soberanía reside en el pueblo que afirma y reivindica su entidad como nación política, y no en el Estado ${ }^{5}$.

Esta profundización cualitativa en la etnicidad que supone la adopción de posiciones nacionalistas se produce especialmente en la época de la transición política, paralelamente a lucha de la mayoría del pueblo catalán por el Estatuto de Autonomía. El contacto con estas luchas provocó que un grupo de andaluces —que ya había tomado conciencia de la existencia de la nación cultural que es Andalucía - dieran un paso más y pasasen a reivindicar el reconocimiento de esta como unidad política diferenciada del Estado, y con ello, el derecho a su existencia como grupo nacional diferenciado allí donde residan.

Esta conciencia y voluntad de formar parte de un pueblo culturalmente definido por unas características específicas y con derecho a ser nación política, al igual que Cataluña, ha tenido que hacer frente al rechazo de amplios sectores de la sociedad catalana. No sólo de aquéllos que niegan la posibilidad de que existan naciones dentro del Estado, sino de aquéllos que aspiran al reconocimiento de Cataluña como nación. Para ambos grupos, la actitud de la minoría nacional andaluza en Cataluña supone un retorno a las posiciones le-

5. Moreno, I.: «Etnicidad, conciencia de etnicidad y movimientos nacionalistas». 
rrouxistas. En concreto, un importante sector del nacionalismo catalán considera que Andalucía no tiene entidad como nación, siendo sólo una región del Estado español. En esta confusión vuelve a jugar un papel fundamental el tema de la lengua. Como Andalucía carece de lengua propia, se le niega la posibilidad de tener una identidad específica y diferenciada del Estado. Aquí actúa la citada confusión entre el vehículo transmisor de la identidad cultural de un pueblo y la propia identidad cultural de éste.

\section{CONCLUSION}

Somos conscientes de las dificultades que acompañan al establecimiento de esta tipología. En primer lugar, las diferencias que separan a las distintas minorías citadas, con ser cualitativamente importantes, no son infranqueables. Tenemos que tener encuenta que la inserción de los andaluces en Cataluña se encuentra sujeta a los procesos de cambio que afectan tanto a la sociedad catalana como a la andaluza y al conjunto de Estado. Así, podemos afirmar que en un primer momento de su inmigración la mayoría de los andaluces pertenecían a la categoría que hemos denominado minoría regional, la reivindicación de su particularidad cultural era prácticamente inexistente y los diversos actos realizados — de carácter regional- eran instrumentalizados por los sectores interesados en frenar la expansión del movimiento nacionalista catalán. Posteriormente, coincidiendo con el auge de las reivindicaciones autonómicas, la mayoría de los andaluces en Cataluña tomaron conciencia de su particularidad étnica. Dos factores influyeron decisivamente en este hecho: por un lado, la incorporación activa de los inmigrantes andaluces a las luchas políticas y sindicales de mediados de los años setenta y por otro, la estancia en Cataluña les había permitido comprobar las diferencias existentes tanto entre andalues y catalanes como entre andaluces y los miembros de otros colectivos de inmigrados, reafirmando de manera decisiva la propia identidad. Paralelamente a este proceso un grupo de andaluces - poco numeroso, pero muy significativo debido a la actividad política que desarrollan - pasaba a constituir una minoría nacional, reivindicando no sólo su identidad étnica sino también el derecho a la soberanía del pueblo andaluz.

Aunque resulta difícil establecer cuáles son las vías concretas que va a seguir el proceso de inserción de los andaluces en la sociedad catalana, hay varios hechos a tener en cuenta que sí pueden adelantarse: en primer lugar, la interdependencia entre este proceso y la coyuntura política y económica estatal, particularmente de Cataluña y - aunque en menor medida- también de Andalucía. Esto significa que un cambio en el gobierno autonómico o estatal, o la superación o continuación de la crisis económica pueden afectar - y de 
hecho afectan - al modelo de inserción de los andaluces en la sociedad catalana. Sin embargo, a pesar de la evidente movilidad que caracteriza al proceso de constitución de las minorías que hemos establecido, tenemos que señalar que, una vez que se ha extendido la autoconciencia de etnicidad hasta el punto de que la mayoría de los andaluces en Cataluña se constituyen en minoría étnica, los intentos de los distintos grupos y sectores interesados en instrumentalizar la etnicidad andaluza para sus propios fines resultan muy delicados y puede que incluso contraproducentes para éstos, pues hemos podido comprobar la decidida voluntad de los andaluces a seguir existiendo como grupo étnico diferenciado pese a todos los intentos integracionistas. En cualquier caso este es un proceso complejo que está prácticamente sin analizar. La validez o no del modelo teórico que proponemos aquí es algo que posteriores estudios y la propia dinámica social se encargarán de demostrar. 